\title{
Structural Parameters and Electronic Structure of Monolayers of Transition Metal Dichalcogenides from $A b$ Initio Calculations
}

\author{
T. WOŹNIAK ${ }^{a, *}$, P. SCHAROCH ${ }^{a}$ AND M.J. WiniARSKI ${ }^{b}$ \\ ${ }^{a}$ Department of Theoretical Physics, Wrocław University of Technology, \\ Wyb. Wyspiańskiego 27, 50-370 Wrocław, Poland \\ ${ }^{b}$ Institute of Low Temperature and Structure Research, Polish Academy of Sciences,
}

Okólna 2, 50-422 Wrocław, Poland

\begin{abstract}
Structural and electronic properties of the $2 \mathrm{H}-\mathrm{MX}_{2}(\mathrm{M}=\mathrm{Mo}, \mathrm{W} ; \mathrm{X}=\mathrm{S}, \mathrm{Se}, \mathrm{Te})$ monolayers are investigated in terms of density functional theory methods. A comparison of structural parameters, energy gaps and spinorbit coupling driven splittings obtained with different pseudopotential methods and various parametrization of exchange-correlation functional is presented.
\end{abstract}

DOI: 10.12693/APhysPolA.129.A-56

PACS: 31.15.A-, 31.15.aj, 61.82.Fk, 73.22.-f

\section{Introduction}

Recently, monolayers of group-VI ${ }^{B}$ transition metal dichalcogenides (TMDs) have attracted significant interest due to their extraordinary electronic and optical properties [1]. Most of them are semiconductors with a direct band gap in the energy range of light, high carrier mobility at room temperature, thermal stability and large intrinsic spin splittings of energy bands, making them promising candidates for optoelectronic and spintronic applications. Their high scalability has already been demonstrated in various devices [2-8].

Bulk TMDs are formed of two-dimensional layers stacked by weak van der Waals forces. The most stable polytype is specified as $2 \mathrm{H}-\mathrm{MX}_{2}$. It has been proven experimentally that decreasing the material thickness to an atomic layer causes a shift from the indirect bulk band gap to a direct one [9]. The lack of the inversion symmetry in single layers allows a significant spin splitting of the bands, due to the strong spin-orbit coupling (SOC) related to heavy atoms. The carriers in the Brillouin zone corners acquire an additional valley degree of freedom, which makes TMDs monolayers possible hosts for optically controlled valleytronic devices [10, 11].

In this work we investigate into the structural and electronic properties of $\mathrm{MoS}_{2}, \mathrm{WS}_{2}, \mathrm{MoSe}_{2}, \mathrm{MoTe}_{2}$, and $\mathrm{WTe}_{2}$ in the framework of DFT-based methods. The results obtained at chosen approximations for exchange-correlation functional and atom representations are compared with the available experimental data.

\section{Computational methods}

The unit cell of $2 \mathrm{H}-\mathrm{MX}_{2}$ is shown in Fig. 1a. A honeycomb layer of $\mathrm{M}$ atoms is sandwiched between two

*corresponding author; e-mail: 184152@student.pwr.edu.pl

$\mathrm{X}$ atomic layers. This hexagonal structure has no inversion center and exhibits $D_{3 h}$ point group symmetry ( $P \overline{6} m 2$ Bravais lattice). The first Brillouin zone of such layered systems is presented in Fig. 1b.

(a)

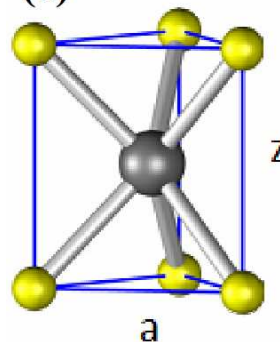

a

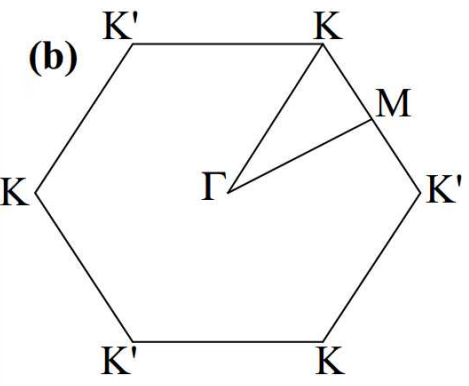

Fig. 1. Unit cell of $\mathrm{MX}_{2}$ (a). Metal $\mathrm{M}$ and chalcogen $\mathrm{X}$ atoms marked grey and yellow, respectively. The related first Brillouin zone (b).

The $a b$ initio calculations were conducted with ABINIT software [12]. The slabs of single TMD layers were separated by vacuum region of more than $15 \AA$. Atoms were modeled in the frame of fully relativistic Hartwigsen-Goedecker-Hutter (HGH) pseudopotentials [13], as well as projector augmented wave (PAW) atomic datasets [14] within the local density (LDA) and general gradient (GGA) approximations of the exchangecorrelation functional $[15,16]$. Furthermore, for band gap calculations the modified Becke--Johnson (MBJLDA) approach [17] was employed basing on the HGH pseudopotentials. Structural parameters were optimized until the interatomic forces were smaller than $5 \times 10^{-5} \mathrm{Ha} / \mathrm{bohr}$. A $10 \times 10 \times 1$ and $24 \times 24 \times 1$ Monkhorst-Pack $k$-point grids were used for structural optimization and band structure calculations, respectively. The plane wave basis cutoff was set to $60 \mathrm{Ha}$ for $\mathrm{HGH}$ and $20 \mathrm{Ha}$ for PAW calculations. 


\section{Results and discussion}

The calculated values of lattice parameters and monolayer thickness are presented in Fig. 2. Generally, the results of fully and scalar relativistic calculations are very close to each other. The GGA and LDA parametrizations of exchange-correlation potential yield over- and underestimated values of lattice parameters, respectively, when compared to the experimental data and being close to results of other ab initio calculations [18-24]. The relatively good agreement between calculated and experimental structural parameters was obtained for the sulfur and tellurium based compounds within all used here approaches whereas in the case of selenium based TMDs the use of PAW datasets leads to a significant overestimation of lattice parameters and the layer thickness. This effect is related to the selection of valence basis sets and the description of semicore atomic states in particular pseudopotentials, which was different in PAW and HGH approaches. Interestingly, an analogous discrepancy between structural parameters obtained from calculations with various pseudopotential methods and experimental studies for selenium and tellurium-based systems was also reported for layered iron chalcogenides [27]. A further discussion of this issue is beyond the scope of this work. It is worth noting that the results of HGH (LDA) calculations are in good agreement with the experimental data for $\mathrm{MoSe}_{2}$ and $\mathrm{WSe}_{2}$.

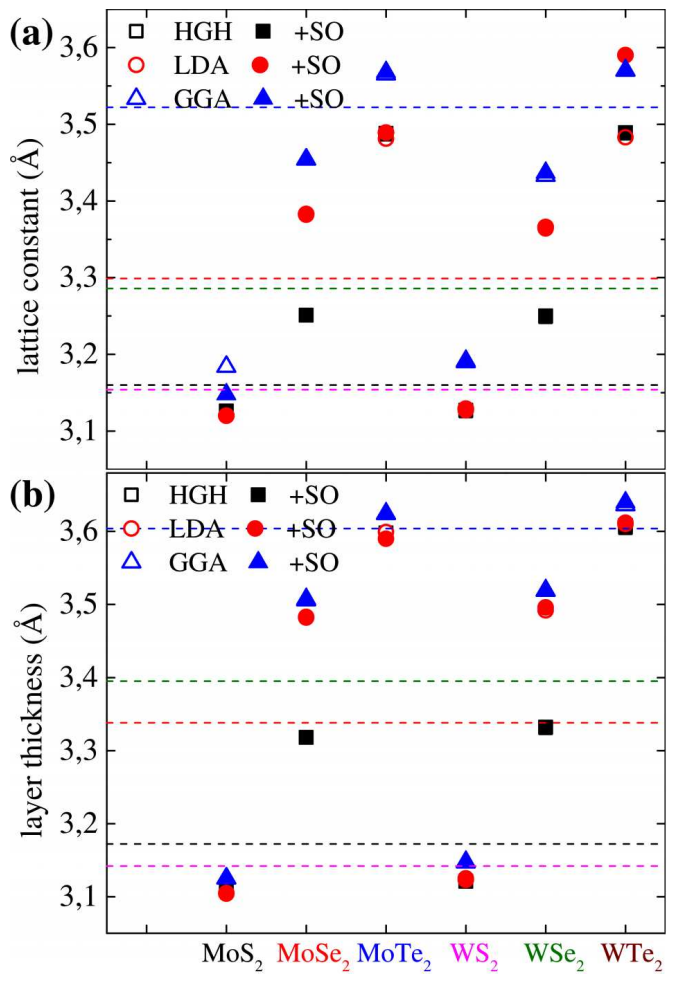

Fig. 2. Calculated in-plane lattice constant (a) and layer thickness (b) obtained with LDA HGH and PAW, as well as GGA PAW pseudopotentials. Unfilled and filled markers correspond to calculations without and with spin-orbit coupling, respectively. Avaible experimental data $[1,9,25,26]$ are marked with colored lines.
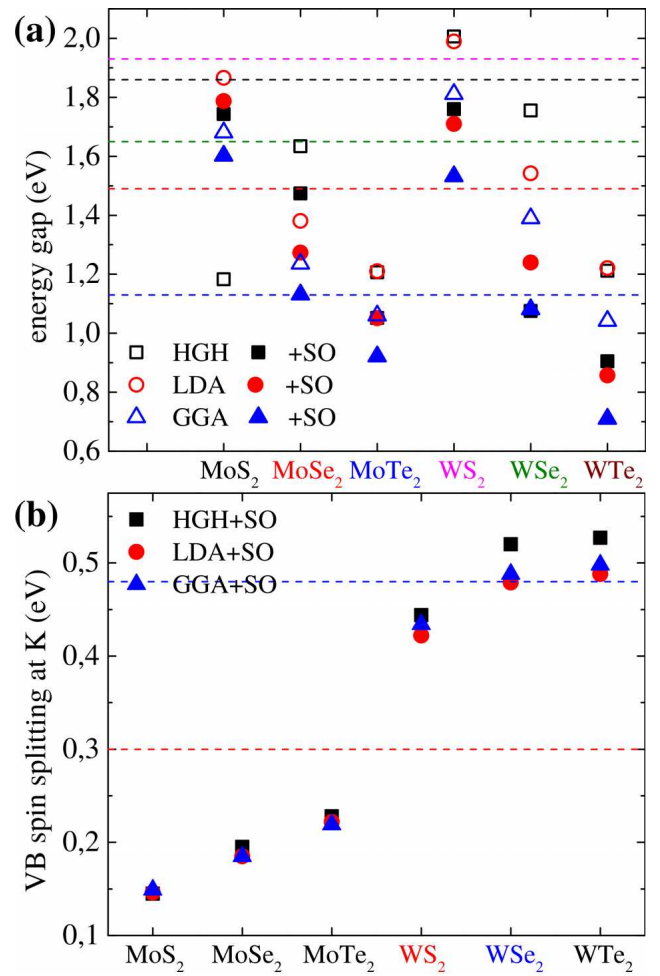

Fig. 3. Calculated with the MBJLDA approach energy gap (a) and spin splitting of valence band in point $K$ (b) related to the structural parameters obtained with LDA HGH and PAW, as well as GGA PAW pseudopotentials. Unfilled and filled markers correspond to calculations without and with spin-orbit coupling, respectively. Avaible experimental data $[1,9,25,26]$ are marked with colored lines.

The calculated values of band gap, presented in Fig. 3a, differ significantly from each other for the particular system due to a selection of exchange-correlation functional, an influence of structural parameters, and an inclusion of SOC. Although the GGA approximation leads to the clear underestimation of band gaps for all studied systems, the LDA results are suprisingly adequate, even when compared to the MBJLDA ones. Such a disparity between GGA and LDA results is probably connected with significantly different structural parameters obtained with both approaches. The standard MBJLDA (HGH) calculations yield unsatisfactory band gaps of studied here TMDs. This effect suggests that the calculations should be rather performed with empirically adjusted value of $c_{\mathrm{M}}$ parameter in the MBJLDA potential [17]. Interestingly, the inclusion of SOC in all calculations (LDA/GGA/MBJLDA) generally decreased the band gaps and do not improved the agreement between our results and experimental data, except the case of $\mathrm{MoSe}_{2}$.

One can expect an enhancement of valence band splitting between Mo- and W-based compounds. As presented in Fig. 3a, the MBJLDA (HGH) calculations leads to slightly higher values of this quantity when compared to the PAW ones. Despite the very good agreement 
between DFT-derived values and the experimental data for $\mathrm{WSe}_{2}$, in the case of $\mathrm{WS}_{2}$ valence band splitting is strongly overestimated. This effect is somewhat unexpected since all methods used here well resemble the structural parameters and the band gap of this system.

\section{Conclusions}

The structural parameters and electronic structure of $2 \mathrm{H}-\mathrm{MX}_{2}(\mathrm{M}=\mathrm{Mo}, \mathrm{W} ; \mathrm{X}=\mathrm{S}, \mathrm{Se}, \mathrm{Te})$ monolayers were investigated with DFT-based fully relativistic calculations. A satisfactory agreement between calculated lattice parameters and experimental data was obtained only for LDA HGH-type pseudopotential method. Meantime, the adequate values of band gaps and valence band splittings for all studied systems were obtained for LDA PAW atomic sets. The standard MBJLDA (HGH) calculations do not improve the agreement between the calculated values of band gaps and the experimental data.

\section{Acknowledgments}

Calculations were performed in Wrocław Centre for Networking and Supercomputing.

\section{References}

[1] Q.H. Wang, K. Kalantar-Zadeh, A. Kis, J.N. Coleman, M.S. Strano, Nat. Nanotechnol. 7, 699 (2012).

[2] B. Radisavljevic, A. Radenovic, J. Brivio, V. Giacometti, A. Kis, Nat. Nanotechnol. 6, 147 (2011).

[3] Z. Yin, H. Li, H. Li, L. Jiang, Y. Shi, Y. Sun, G. Lu, Q. Zhang, X. Chen, H. Zhang, ACS Nano 6, 74 (2011).

[4] H.S. Lee, S.W. Min, Y.G. Chang, M.K. Park, T. Nam, H. Kim, J.H. Kim, S. Ryu, S. Im, Nano Lett. 12, 3695 (2012).

[5] B. Radisavljevic, M.B. Whitwick, A. Kis, Appl. Phys. Lett. 101, 043103 (2012).

[6] F.K. Perkins, A.L. Friedman, E. Cobas, P.M. Campbell, G.G. Jernigan, B.T. Jonker, Nano Lett. 13, 668 (2013).

[7] O. Lopez-Sanchez, D. Lembke, M. Kayci, A. Radenovic, A. Kis, Nat. Nanotechnol. 8, 497 (2013).

[8] M. Bernardi, M. Palummo, J.C. Grossman, Nano Lett. 13, 3664 (2013).
[9] K.F. Mak, Ch. Lee, J. Hone, J. Shan, T.F. Heinz, Phys. Rev. Lett. 105, 136805 (2010).

[10] H. Zeng, J. Dai, W. Yao, D. Xiao, X. Cui, Nat. Nanotechnol. 7, 490 (2012).

[11] K.F. Mak, K. He, J. Shan, T.F. Heinz, Nat. Nanotechnol. 7, 494 (2012).

[12] X. Gonze, B. Amadon, P.M. Anglade, J.M. Beuken, F. Bottin, P. Boulanger, F. Bruneval, D. Caliste, R. Caracas, M. Cote, T. Deutsch, L. Genovese, Ph. Ghosez, M. Giantomassi, S. Goedecker, D.R. Hamann, P. Hermet, F. Jollet, G. Jomard, S. Leroux, M. Mancini, S. Mazevet, M.J.T. Oliveira, G. Onida, Y. Pouillon, T. Rangel, G.M. Rignanese, D. Sangalli, R. Shaltaf, M. Torrent, M.J. Verstraete, G. Zerah, J.W. Zwanziger, Comput. Phys. Commun. 180, 2582 (2009).

[13] C. Hartwigsen, S. Goedecker, J. Hutter, Phys. Rev. B 58, 3641 (1998).

[14] N.A.W. Holzwarth, A.R. Tackett, G.E. Matthews, Comput. Phys. Commun. 135, 329 (2001).

[15] J.P. Perdew, Y. Wang, Phys. Rev. B 45, 13244 (1992).

[16] J.P. Perdew, K. Burke, M. Ernzerhof, Phys. Rev. Lett. 77, 3865 (1996).

[17] F. Tran, P. Blaha, Phys. Rev. Lett 102, 226401 (2009).

[18] Y. Ding, Y. Wang, J. Ni, L. Shi, S. Shi, W. Tang, Physica B 406, 2254 (2011).

[19] S. Yun, S.W. Han, S.C. Hong, I.G. Kim, J.D. Lee, Phys. Rev. B 85, 033305 (2012).

[20] C. Ataca, H. Sahin, S. Ciraci, J. Phys. Chem. C 116 , 8993 (2012).

[21] H. Jiang, J. Phys. Chem. C 116, 7664 (2012).

[22] P. Johari, V.B. Shenoy, ACS Nano 6, 5449 (2012).

[23] G.B. Liu, W.Y Shan, Y. Yao, W. Yao, D. Xiao, Phys. Rev. B 88, 085433 (2013).

[24] Kang, S. Tongay, J. Zhou, J. Li, J. Wu, Appl. Phys. Lett. 102, 012111 (2013).

[25] L. Liu, S.B. Kumar, Y. Ouyang, J. Guo, IEEE Trans. Electron Dev. 58, 3042 (2011).

[26] D. Wickramaratne, F. Zahid, R.K. Lake, J. Chem. Phys. 140, 124710 (2014).

[27] M.J. Winiarski, M. Samsel-Czekała, A. Ciechan, J. Appl. Phys. 116, 223903 (2014). 